\title{
Beyond First-Line Therapy: Combining Chemotherapy and Radioembolization for Hepatic Colorectal Metastases
}

\author{
Joshua E. Meyer and Steven J. Cohen*
}

Department of Medical Oncology, Fox Chase Cancer Center, 333 Cottman Avenue Room C303 Philadelphia, PA

\begin{abstract}
Metastatic colorectal cancer (mCRC) remains largely an incurable condition. Although progress has been made in expanding the number of available systemic agents, treatment after initial therapy has limited benefit. Radioembolization (RE) with radiolabeled microspheres is an emerging treatment modality for mCRC. Given the potential benefit of chemotherapy to control systemic disease and act as a radiosensitizer, there is high enthusiasm for studying RE and chemotherapy combinations, particularly after initial systemic therapy for patients with liver dominant or confined disease. This manuscript reviews the rationale for this approach, summarizes recently reported clinical trials, and provides a perspective for everyday practice and future unanswered research questions.
\end{abstract}

\section{Introduction}

Colorectal cancer remains a significant cause of cancer related mortality, with over 140,000 new cases and 50,000 deaths in the United States each year [1]. Recent advances in the treatment of metastatic colorectal cancer (mCRC) have improved median survival to over 2 years [2]. However, the majority of benefit results from initial (firstline) chemotherapy. Treatment benefit beyond first-line therapy is more modest, with response rates of less than twenty percent and survival benefit of at most 2-4 months [3]. Radiation therapy is a standard component of treatment for localized rectal cancer. With concurrent chemotherapy, approximately half of patients have tumor downstaging and pathologic complete response rates are $15-25 \%$ [4]. Thus, there is interest in incorporating radiotherapy in other disease scenarios within the colorectal cancer spectrum, such as liver metastases. Radioembolization (RE) with radiolabeled microspheres is one such modality. Historically, the literature supporting RE for colorectal cancer liver metastases has primarily included retrospective reviews and case series, with encouraging response rates. More recently, larger prospective series and randomized trials have demonstrated a benefit. Building upon the rationale for chemotherapy radiosensitization noted in the treatment of rectal cancer, combining systemic chemotherapy and RE is an attractive treatment strategy, particularly beyond firstline systemic therapy where benefits of chemotherapy alone are more modest. The current review will first give an overview of systemic chemotherapy utilized for metastatic colorectal cancer after initial treatment (i.e. second line and beyond). We will then review evidence for combining RE with chemotherapy in refractory mCRC. This will include the initial results from our center's experience in a phase 1 combination study of capecitabine and RE. Finally, we will discuss our everyday practice and unanswered questions for future study.

\section{Systemic therapy options for metastatic colorectal cancer}

First-line: Treatment for mCRC has historically involved chemotherapy alone. In 2011, there are five classes of agents available for use. As initial therapy, the most common strategy is to combine a fluoropyrimidine (infusional 5-Fluorouracil or capecitabine) with either oxaliplatin or irinotecan. The resultant combinations with infusional 5-FU (FOLFOX or FOLFIRI) have been compared to each other as first-line therapy and yield similar outcomes [5]. Approximately $50 \%$ of patients have objective tumor responses and median survival is approximately 2 years. The combination of capecitabine with oxaliplatin (Capox, Xelox) is similar in efficacy to FOLFOX [6], although the combination of capecitabine with irinotecan (Capiri) is generally felt to be too toxic for routine use [7].

In addition to chemotherapy, the incorporation of a monoclonal antibody improves first-line outcome further. Bevacizumab, an anti-vascular endothelial growth factor antibody, has demonstrated improved survival when combined with first-line chemotherapy [8] Cetuximab and panitumumab, anti-epidermal growth factor inhibitor antibodies, also improve outcome with first-line chemotherapy [2,9], although their incorporation has generally been reserved for secondand third-line treatment $[10,11]$. Thus, the vast majority of newly diagnosed patients with $\mathrm{mCRC}$ in the United States initially receive FOLFOX plus bevacizumab.

Beyond first-line: As oxaliplatin-based therapy emerged as the preferential initial treatment, irinotecan-based therapy has predominantly been utilized in second-line and beyond. Recent results from second-line trials have indicated that efficacy beyond FOLFOX is quite limited, with irinotecan alone resulting in response rates of less than 5\% [3]. The addition of cetuximab for patients with KRAS wild-type tumors improves response rates to $20 \%$, but median time to progression is only 4 months [11]. Patients who receive irinotecan without an antibody as second-line treatment may have the antibody added for third line treatment. Alternatively, patients receiving irinotecan-based initial therapy may have FOLFOX utilized as thirdline treatment. However, response rates and outcomes remain poor.

Rationale for liver-directed strategies: The liver remains the most common site of metastases in colorectal cancer, with $60 \%$ of patients with metastatic disease having liver involvement [12]. Although complete resection of metastatic disease remains the gold standard for potential cure, this is only attempted in $5-10 \%$ of those with liver metastases. Thus, for most mCRC patients, progression of

*Corresponding author: Steven J. Cohen, Department of Medical Oncology, Fox Chase Cancer Center, 333 Cottman Avenue Room C303 Philadelphia, PA 191112497, E-mail: steven.cohen@fccc.edu

Received April 26, 2011; Accepted May 26, 2011; Published June 15, 2011

Citation: Meyer JE, Cohen SJ (2011) Beyond First-Line Therapy: Combining Chemotherapy and Radioembolization for Hepatic Colorectal Metastases. J Nucl Med Radiat Ther 2:103. doi:10.4172/2155-9619.1000103

Copyright: (C) 2011 Meyer JE, et al. This is an open-access article distributed under the terms of the Creative Commons Attribution License, which permits unrestricted use, distribution, and reproduction in any medium, provided the original author and source are credited. 
liver metastases remains the leading cause of death. As normal liver derives most of its blood supply from the portal circulation and liver metastases derive most of their blood supply from the hepatic artery, several different liver-directed strategies have been evaluated. Intrahepatic delivery of fluoropyrimidine chemotherapy via indwelling pump is perhaps the modality with the longest, best supported dataset, with several single arm and randomized studies having been reported. Compared to systemic fluoropyrimidine, intrahepatic delivery results in increased response rates in liver [13]. Long-term followup demonstrates improved survival for mCRC patients treated with intrahepatic compared to systemic fluoropyrimidine [14]. In addition, two adjuvant trials after liver resection have demonstrated improved outcome with utilization of intrahepatic fluoropyrimidine [12,15]. However, implantation of a hepatic artery infusion pump requires surgery, and experience is required to avoid complications such as biliary toxicity. In addition, the availability of increased numbers of systemic agents has tempered enthusiasm for this approach.

Radiolabeled microspheres: Radiolabeled microspheres are an emerging treatment modality. This technique similarly takes advantage of the differential blood supply to liver metastases $(80-100 \%$ blood supply from the hepatic artery) as compared to the normal liver tissue (70-80\% blood supply from the portal vein) [16]. This allows the delivery of high doses of radiation to liver tumors, while sparing the normal tissue from the majority of the associated toxicity. While the maximum safe dose of radiation to the whole liver delivered with external beam radiation therapy is only 30-35 Gy, much higher doses are necessary in order to control metastatic disease in the liver [17]. While higher doses are possible utilizing such techniques as brachytherapy or stereotactic body radiation therapy, they are unable to address the common clinical presentation of liver metastases with multiple nodules extending across one or both lobes $[18,19]$. However, higher doses can be delivered safely in this setting with RE, utilizing the differential blood supply and, in effect, microtargeting the tumors [20].

There are two radiolabeled microsphere products currently available. The first is SIR-Spheres, made of resin, 35 microns in diameter, with an activity of $50 \mathrm{~Bq}$ per particle. The second is TheraSpheres, made of glass, 25 microns in diameter, with an activity of $2500 \mathrm{~Bq}$ per particle. Both use yttrium-90 as the isotope bound to the sphere that delivers the radiation. Yttrium-90 has a mean tissue penetration of $2.5 \mathrm{~mm}$ and a maximum of $11 \mathrm{~mm}$, with a half-life of 2.7 days [21].

The process of radioembolization (also called selective internal radiation therapy) is a multidisciplinary collaboration often including interventional radiologists, radiation oncologists, and nuclear medicine physicians in order to perform the procedure safely and effectively. Important pieces of the process include initial consultation, staging arteriogram, and technetium-labeled macroaggregated albumin scan to assess lung shunt, dose calculation, and delivery [22]. These have been recently reviewed and summarized [16].

\section{Combining chemotherapy and radioembolization}

Rationale: There are several concepts which support combining systemic chemotherapy and RE. For one, although most patients with $\mathrm{mCRC}$ and liver involvement die of progressive liver failure, improving systemic therapy has resulted in an increasing incidence of extrahepatic metastases. In our experience, with greater longevity comes an increasing incidence of metastases in unusual locations such as bone and brain [23]. Thus, combining systemic and liver-directed strategies can control both intra- and extra-hepatic disease. Secondly, there is potential for a radiosensitizing effect of systemic therapy with combination approaches. Throughout the gastrointestinal tract in general $[24,25]$ and described above in colorectal cancer in particular [4], emerging data suggest that combination chemoradiotherapy programs improve outcome and enhance local control. It stands to reason that this may be true of intrahepatic CRC metastases.

Prospective studies: Historically, the database supporting the efficacy of RE either alone or in combination with chemotherapy has consisted of retrospective reports. More recently, well designed prospective trials evaluating the safety and efficacy of concurrent chemotherapy and radioembolization have been reported (Table 1). A phase I dose escalation study of irinotecan with Yttrium-90 resin microsphere RE was recently published by van Hazel and colleagues [26]. This study enrolled patients at four centers in Australia with mCRC who had progressed on prior 5-FU-based chemotherapy and harbored either liver-only or liver-dominant disease. A standard $3+3$ design was utilized, with irinotecan administered at escalating doses of 50,75 , or $100 \mathrm{mg} / \mathrm{m} 2$ on days 1 and 8 every 21 days for two cycles. This was followed by seven cycles of irinotecan at $100 \mathrm{mg} / \mathrm{m} 2$. RE was performed on day 2 or 3 of cycle 1 to the entire liver.

Twenty-five patients were treated in total. Two-thirds of patients received one prior chemotherapy regimen and one-third 2-3. The median delivered dose of RE was $1.80 \mathrm{GBq}$. An initial cohort of patients received irinotecan at $50 \mathrm{mg} / \mathrm{m}^{2}$ weekly for 4 weeks followed by 2 weeks rest. This older irinotecan treatment approach has generally resulted in increased toxicity and is not utilized today. In the current trial, of three patients treated at this dose level, one had an abscess and one intolerable diarrhea and thus the protocol was amended to the days 1 and 8 every 21 days schedule. At these dose levels, dose-limiting toxicity as defined in the protocol (an increase of $25 \%$ in grade $3 / 4$ toxicity over that expected from irinotecan alone) was not experienced at any dose level and thus the maximum tolerated dose was not reached. Transient changes in serum bilirubin and albumin, but no significant increases of liver enzymes were seen. One patient developed a grade 2 gastric ulcer. Eleven patients experienced mild abdominal pain and 14 patients experienced nausea.

Of 23 patients evaluable for efficacy, eleven patients (48\%) had a partial response and nine patients (39\%) had stable disease as their best response. One patient ultimately had a complete response and remains

\begin{tabular}{|c|c|c|c|c|c|c|c|}
\hline Author & Study type & $\mathbf{N}$ & Chemotherapy & RE & Disease Control* (\%) & Median PFS (months) & Toxicity** (\%) \\
\hline Van Hazel & Dose escalation & 25 & Irinotecan $\left(50-100 \mathrm{mg} / \mathrm{m}^{2}\right)$ & Whole liver & 87 & 6.0 & 28 \\
\hline Lim & Prospective registry & 30 & Bolus 5-FU & Whole liver & 60 & 5.3 & 23 \\
\hline Hendlisz & Phase III & $\begin{array}{l}23 \\
23\end{array}$ & $\begin{array}{l}\text { 5-FU }\left(225-300 \mathrm{mg} / \mathrm{m}^{2}\right) \\
5-\mathrm{FU}\left(300 \mathrm{mg} / \mathrm{m}^{2}\right)\end{array}$ & $\begin{array}{l}\text { Whole liver } \\
\text { N/A }\end{array}$ & $\begin{array}{l}86 \\
35\end{array}$ & $\begin{array}{l}4.5 \\
2.1\end{array}$ & $\begin{array}{l}4 \\
26\end{array}$ \\
\hline Chua & Case series & 140 & $5-F U 225 \mathrm{mg} / \mathrm{m}^{2}$ & Involved lobe(s) & 63 & NR & NR \\
\hline Cohen & Dose escalation & 21 & Capecitabine $\left(375-1000 \mathrm{mg} / \mathrm{m}^{2}\right)$ & Lobar & 95 & $N R^{*}$ & 17 \\
\hline
\end{tabular}

*Defined as sum of percentage of patients with complete response, partial response, or stable disease

**Defined as either grade $3 / 4$ or serious non-hematological toxicity

Table 1: Summary of combination radioembolization and chemotherapy trials for refractory metastatic colorectal cancer. 
free of disease 5 years from subsequent RFA for a single localized recurrent lesion. Median progression-free survival (PFS) was 6.0 months, with median PFS in the liver of 9.2 months. Median overall survival was 12.2 months. As in other studies, patients with liver-only disease had the longest survival. Although cross-study comparisons should always be viewed with caution given differing patient selection criteria, these efficacy results compare quite favorably to recent studies of irinotecan therapy alone in second-line treatment, where response rates are generally less than $10 \%$ and PFS 4 months.

A second prospective study of chemotherapy in combination with RE for patients with previously treated mCRC was reported by Lim et al and similarly conducted at several Australian centers [27]. Thirty patients who had liver-only or liver-dominant disease and had previously been treated and progressed on 5-FU-based chemotherapy were enrolled. One-quarter of patients had received only 5-FU and nearly 50\% 5-FU, irinotecan, and oxaliplatin. All patients were treated with RE and concurrent bolus 5-FU, although chemotherapy dosing was not reported.

Toxicity in this study was not as clearly reported as the van Hazel report above, but mostly consisted of mild lethargy, anorexia, nausea, and right upper quadrant pain in the weeks following procedure. However, there were some serious toxicities noted. One patient presented shortly after treatment with worsening liver enzymes, liver failure and death. This was felt likely related to disease progression by the investigators. Additionally, four patients developed gastric or duodenal ulcers. The authors comment that the routine administration of acid suppressive medications was not part of their protocol, but should be recommended in the future. One patient developed acute right upper quadrant pain and elevation of liver enzymes that required hospital admission and resolved with conservative management.

Ten patients (33\%) exhibited a partial response, including one patient who eventually demonstrated a complete response to therapy. An additional 8 patients demonstrated stable disease as best response. Interestingly, continued reduction in the size of lesions was seen up to 1 year after therapy. Seven of the patients had extra-hepatic (but liverdominant) disease, and none of these patients demonstrated a response. The median time to progression was 5.3 months. As 5-FU has essentially a zero percent response rate after prior progression, these efficacy data compare favorably to chemotherapy alone.

While provocative, single arm trials can only provide initial evidence of treatment benefit and randomized trials are required to better define the contribution of chemotherapy to RE treatment. In 2010, Hendlisz and colleague published the only phase III study results of chemotherapy and radiolabeled microspheres in refractory mCRC [28]. All 46 patients had received 5-FU, irinotecan, and oxaliplatin and were either intolerant of or had progressed on all. All patients had liver confined disease and were randomized to either receive (1) 5-FU alone $\left(300 \mathrm{mg} / \mathrm{m}^{2}\right)$ days 1 through 14 every three weeks until progression or (2) 5 -FU $\left(225 \mathrm{mg} / \mathrm{m}^{2}\right)$ days 1 through 14 plus RE for the first cycle followed by 5 -FU $\left(300 \mathrm{mg} / \mathrm{m}^{2}\right)$ days 1 through 14 every three weeks until progression. Radioembolization was performed to the entire liver, with a median dose of $1.79 \mathrm{GBq}$. The trial utilized time to liver progression (TTLP) as the primary endpoint, with approximately $90 \%$ power to detect an increase from 6 weeks in the 5-FU alone arm to 18 weeks in the combination arm. The trial was initially slated to enroll 58 patients but due to slow accrual 46 were enrolled.

Despite the lower than anticipated sample size, patient characteristics including age and ECOG performance status were well balanced between the arms. Grade 3 or 4 toxicity was seen in only one RE patient, versus six patients in the 5-FU alone arm. There were no radiographic responses in the 5-FU alone arm, and only two (9.5\%) in the combination arm. However, the disease control rate (defined as stable disease or partial response) was $35 \%$ and $86 \%$ in the chemotherapy only and RE arms, respectively $(\mathrm{P}=0.001)$. The median time to liver progression was 2.1 and 5.5 months, in favor of the RE $\operatorname{arm}(\mathrm{P}=0.003)$. Median time to progression at any site were 2.1 and 4.5 months, in favor of the RE arm $(\mathrm{P}=0.03)$. Median overall survival was not statistically different between the two arms (7.3 for chemotherapy alone and 10.0 months in the combination, $\mathrm{P}=0.80$ ), which may reflect the fact that $10 / 23$ patients in the chemotherapy alone arm ultimately received $\mathrm{RE}$.

Taken in sum, the three published prospective experiences combining chemotherapy and radiolabeled microspheres demonstrate a toxicity profile consistent with $\mathrm{RE}$ microspheres therapy alone. Efficacy results are suggestive of improvement compared to what would be expected from chemotherapy alone in refractory disease. However, further randomized trial evaluation is required to better delineate the contribution of chemotherapy in this setting.

A large prospectively collected case series of 140 patients with colorectal liver metastases has been reported by Chua and colleagues, in which $94 \%$ of patients had received at least one prior chemotherapy regimen [29]. Although a case series, inclusion criteria were fairly uniform. Patients were required to have liver-only mCRC (limited extrahepatic metastases at one site permitted), good performance status (ECOG 0-2), and serum bilirubin $\leq 2.0 \mathrm{mg} / \mathrm{dL}$ ). Forty-eight patients $(34 \%)$ received concurrent or immediate post-RE systemic chemotherapy with 5-FU, administered as a standard dose 7 day continuous infusion at $225 \mathrm{mg} / \mathrm{m}^{2} /$ day beginning one day prior to $\mathrm{RE}$ . Patients with bilobar disease had both lobes treated concurrently. RE was delivered with a median dose of $1.8 \mathrm{GBq}$.

No treatment related deaths were reported. Approximately one quarter of patients had early toxicity from treatment, consisting primarily of abdominal pain (14\%) and nausea (5\%). Three patients had gastritis and one had intestinal ulceration. Seven (5\%) patients had late effects - three with radiation-induced liver dysfunction, four with intestinal ulceration, and one with gallbladder/biliary complications. The authors did not specifically address whether these complications occurred in patients receiving concurrent chemotherapy.

Patients treated with concurrent chemotherapy demonstrated a complete or partial response by RECIST criteria of $48 \%$, compared to $24 \%$ in those receiving $\mathrm{RE}$ alone. Univariate analysis identified the addition of chemotherapy as predictive of treatment response $(\mathrm{P}=0.007)$, and multivariate analysis confirmed concurrent chemotherapy as the only predictive factor for a favorable (complete or partial) response $(\mathrm{P}=0.007)$. Patients receiving chemotherapy also had longer overall survival in univariate analysis ( 7 versus 13 months, $\mathrm{P}=0.017$ ), but this difference did not persist in multivariate analysis. Median overall survival was 9 months for the entire cohort.

Comparison of the various chemotherapy and RE regimens with one another is difficult for a number of reasons. Most of these studies include small numbers of patients, and those patients are heterogeneous in terms of burden of disease, performance status, and number of prior chemotherapy regimens. Additionally, the methods and timing of response assessment in these studies are quite variable. However, the overall response rates appear encouraging without an increase in toxicity beyond that expected with either chemotherapy or RE alone. 
(A)

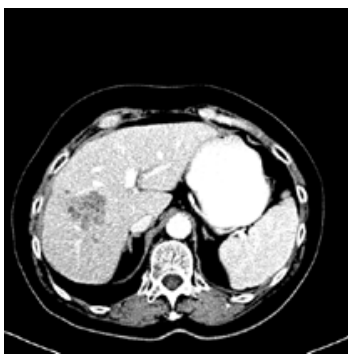

(B)

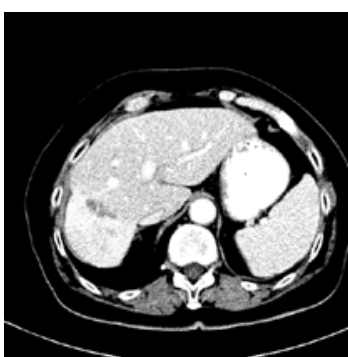

Figure 1: Response of liver metastases in a patient with metastatic colorecta cancer treated on a phase 1 combination study of radiolabeled microspheres and capecitabine.

Fox chase phase 1 study with capecitabine: Given intriguing preliminary evidence of synergy between chemotherapy and radiolabeled microspheres and concerns about toxicity with capecitabine arising from an isolated safety report, we began a dose escalation study of capecitabine in combination with Sir-Spheres in patients with advanced solid tumors [30]. Eligibility criteria were intentionally broad for a phase 1 and included patients with any incurable solid tumor who had received any number of prior therapies. Patients had to have measurable liver metastases, but could also have extrahepatic disease. Patients were required to have excellent liver function (total bilirubin $\leq$ $\mathrm{ULN}$ and ALT/AST $\leq 1.5 \mathrm{X}$ ULN) and have ECOG performance status of 0-1 (fully functional with no or mild tumor-related symptoms). No evidence of portal vein thrombosis was permitted. Once enrolled, patients had to have $<20 \%$ shunting by Macroaggregated Albumin (MAA) Scan.

Patients were enrolled into cohorts of 3-6 patients in a classic $3+3$ phase 1 design. Briefly, patients were enrolled in groups of 3 and toxicity monitored for up to 8 weeks. In the absence of dose-limiting toxicity (DLT), 3 patients were enrolled in the next highest cohort. If $1 / 3$ patients experienced DLT, 3 additional patients were enrolled to that dose level. If $2-3 / 3$ patients had DLT, enrollment was deescalated one dose level below. Dose-limiting toxicity was defined during the first eight weeks as any $\geq$ grade 3 non-hematologic toxicity (excluding nausea/vomiting responding to symptomatic measures), $\geq$ grade 3 thrombocytopenia or $\geq$ grade 4 neutropenia lasting at least 5 days, or any interruption in therapy lasting at least 14 days.

$\mathrm{RE}$ in the form of Sir-Spheres was administered to one lobe of the liver at a time and was determined by treating the lobe with the highest disease burden. The dose of SIR-Spheres in GBq was calculated by the standard formula $=\{(\mathrm{BSA}-0.2)+(\%$ tumor involvement of lobe to be treated/100)\} X \{Estimated percent of total liver that treated lobe comprises\}. Capecitabine was administered at increasing dosing per cohort: $375,600,750,900$, and $1,000 \mathrm{mg} / \mathrm{m}^{2}$ twice daily for 14 days followed by one week rest, beginning the day before Sir-Spheres administration. This 14 day cycle of capecitabine dosing was repeated twice followed by CT disease evaluations at 6-8 weeks. Patients with unilobar disease could receive additional capecitabine at the discretion of the treating physician. Patients with bilobar disease could receive a dose of Sir-Spheres to the contralateral lobe with two additional cycles of capecitabine beginning between weeks $8-10$, assuming all laboratory parameters had returned to eligibility criteria levels.

To date, 21 patients have been enrolled into 4 cohorts. The median age of the patient population is 61 (range 49-81) with 13 men and 8 women. The majority of patients (16) had mCRC, with one each having biliary, hepatocellular, unknown primary, neuroendocrine, and small bowel cancers. Patients had received a median of two prior systemic therapies. At the first dose level (capecitabine $=375 \mathrm{mg} / \mathrm{m}^{2}$ twice daily), of the first 3 patients, one with biliary cancer had grade 3 hyperbilirubinemia conservatively judged as possibly related to treatment. Of the next two patients, one with CRC had grade 4 hyperbilirubinemia judged related to progressive disease. The cohort was expanded to eight patients with no further DLTs. Thus, four patients were enrolled into cohort $2\left(600 \mathrm{mg} / \mathrm{m}^{2}\right.$ twice daily, one patient erroneously took the incorrect capecitabine dose and was replaced). No DLTs were observed. In cohort $3\left(750 \mathrm{mg} / \mathrm{m}^{2}\right)$, one patient with $\mathrm{mCRC}$ and a large left lobe lesion had a partial antral perforation and the cohort was expanded to 6 , with no further DLTs. In cohort $4\left(900 \mathrm{mg} / \mathrm{m}^{2}\right)$ no DLTs were observed in three enrolled patients. Enrollment currently continues in cohort $5\left(1,000 \mathrm{mg} / \mathrm{m}^{2}\right)$. No other grade $3 / 4$ non-hematologic toxicities were noted. Common grade $1 / 2$ non-hematologic toxicities included transaminitis/alkaline phosphatase elevation (9), nausea (8), pain (7), fatigue (5), and hand/foot (5). Although antitumor activity was not the primary endpoint of this trial, best response included confirmed partial response in three patients, unconfirmed partial response in one patient, stable disease in 16 patients, and progressive disease in one patient. A significant response in a mCRC patient is demonstrated in Figure 1.

On balance, our trial to date has demonstrated that capecitabine can be safely combined with RE in the form of Sir-Spheres in patients with advanced cancer with predominantly mCRC. Treatment was generally well tolerated. The one toxicity which requires closer inspection is the partial antral perforation. This patient with $\mathrm{mCRC}$ had a large left sided liver mass. Whether this ulceration occurred due to local radiation effects or deposition of spheres aberrantly in the stomach is unclear The contribution of concurrent capecitabine to this toxicity is also not known. Given the refractory nature of this patient population, the partial responses and stable disease noted is quite encouraging for future study. An expansion cohort in mCRC patients at the MTD is planned.

\section{Everyday practice and future directions}

The literature to date supports the efficacy of radiolabeled microspheres as RE in refractory mCRC patients and suggests that concurrent chemotherapy can be added safely. There are no current randomized trials to delineate whether concurrent chemotherapy improves efficacy compared to RE alone. Ongoing frontline trials with oxaliplatin-containing chemotherapy with or without SirSpheres will hopefully shed additional light on whether RE adds to chemotherapy [31]. Pending these results, in our practice we tend to administer chemotherapy alone to patients with newly diagnosed and $2^{\text {nd }}$-line mCRC patients, given the clear survival benefit associated with these approaches. For patients with refractory disease after standard chemotherapy who have liver-only or dominant disease, we consider treatment with radiolabeled microspheres. We usually administer radiolabeled microspheres alone, as concurrent chemotherapy has not yet been clearly demonstrated to offer an efficacy or survival advantage. Additionally, we deliver therapy to a single lobe, restage, and then treat 


\begin{tabular}{|c|}
\hline Patient selection \\
\hline Optimal systemic therapy "partner" \\
\hline Sequence of treatment \\
\hline Optimal microsphere \\
\hline Optimal clinical endpoint \\
\hline
\end{tabular}

Table 2: Summary of future research questions regarding the combination of radiolabeled microspheres and chemotherapy.

the other lobe if it continues to be clinically indicated (at least stable disease in treated lobe and no significant progression outside the liver).

There are many research questions still to be answered regarding concurrent chemotherapy and radiolabeled microspheres in refractory mCRC. A summary of categories for future research is listed in Table 2. The largest potential category revolves around choice of patient and therapy. For one, which patients are likely to benefit? Will these be patients who initially responded to similar systemic chemotherapy? Or, is the concurrent systemic chemotherapy for refractory patients simply for use as a radiosensitizer and prior response irrelevant? Which chemotherapy regimen should be utilized? Is there an advantage to radiosensitization with oxaliplatin- compared to irinotecan-containing regimens? What should be the optimal role for monoclonal antibodies? Would combining anti-EGFR therapy result in enhanced radiosensitization? Does prior bevacizumab make subsequent radiolabeled microspheres more challenging by altering the vasculature? Or, should bevacizumab be included with concurrent chemotherapy and RE?

A second general category for future research revolves around the optimal sequence of chemotherapy and RE? If administered "concurrently", should chemotherapy begin at the same time as RE? A day before? One week before? In more recent reports in rectal cancer trials, the exact regimen of fluoropyrimidine with radiotherapy has appeared to be of less significance. If chemotherapy and RE are administered concurrently, should chemotherapy be continued beyond RE? If so, for what duration? In our phase 1 trial described above, we empirically began capecitabine the day before Sir-Spheres to maximize radiosensitization. This may or may not be optimal.

Other unanswered questions revolve around the RE itself in combination with chemotherapy. With two products now available in many countries, there are a number of questions that arise regarding the proper use of each modality. The Sir-Spheres radioembolization may be most effective for certain patients, while Theraspheres may offer advantages for others. There are also unanswered questions about the volume of liver that is best treated at once. Not only are there varying practices regarding whole versus lobar therapy, but there exists the possibility of performing sublobar or segmental therapy in the appropriate clinical situation. The volume of liver treated may impact the tolerability of the combination with chemotherapy, just as the size of an external beam radiation therapy field has been shown to impact toxicity in standard chemoradiation.

Finally, from the clinical trialist's perspective, the optimal endpoint to utilize for these studies can be debated. Historically, phase 2 trials in mCRC have utilized time to progression or progression-free survival. This may not be ideal, however, when considering liver-directed RE. For example, if a patient has a dramatic response in liver but develops a single new small lung nodule, this would technically be considered progression. However, this may not be an ideal test of a novel liverdirected treatment strategy. Time to liver progression may better capture the therapeutic impact but this has not been as well adopted by the oncology community as a legitimate endpoint. Future clinical trials should ideally capture both, in order to assess the ideal surrogate for overall survival, the "gold standard" clinical endpoint.

\section{Conclusion}

In summary, increasing clinical trial data suggest that the combination of chemotherapy and radiolabeled microsphere RE can be administered safely to patients with refractory mCRC. Whether this approach improves outcome compared to either modality alone is the subject of ongoing clinical trial efforts. If the combination is indeed proven to improve clinical outcome, numerous other clinical questions regarding duration, sequencing, and particular systemic regimens await future well designed clinical trials.

\section{References}

1. Jemal A, Siegel R, Xu J, Ward E (2010) Cancer Statistics, 2010. CA Cancer J Clin 60: 277-300

2. Van Cutsem E, Köhne CH, Láng I, Folprecht G, Nowacki MP, et al. (2011) Cetuximab Plus Irinotecan, Fluorouracil, and Leucovorin As First-Line Treatment for Metastatic Colorectal Cancer: Updated Analysis of Overall Survival According to Tumor KRAS and BRAF Mutation Status. J Clin Oncol 29: 2011-2019.

3. Sobrero AF, Maurel J, Fehrenbacher L, Scheithauer W, Abubakr YA, et al (2008) EPIC: phase III trial of cetuximab plus irinotecan after fluoropyrimidine and oxaliplatin failure in patients with metastatic colorectal cancer. J Clin Oncol 26: 2311-2319

4. Braendengen M, Tveit KM, Berglund A, Birkemeyer E, Frykholm G, et al (2008) Randomized phase III study comparing preoperative radiotherapy with chemoradiotherapy in nonresectable rectal cancer. J Clin Oncol 26: $3687-$ 3694.

5. Tournigand C, André T, Achille E, Lledo G, Flesh M, et al. (2004) FOLFIR followed by FOLFOX6 or the reverse sequence in advanced colorectal cancer: a randomized GERCOR study. J Clin Oncol 22: 229-237.

6. Cassidy J, Clarke S, Díaz-Rubio E, Scheithauer W, Figer A, et al. (2008) Randomized phase III study of capecitabine plus oxaliplatin compared with fluorouracil/folinic acid plus oxaliplatin as first-line therapy for metastatic colorectal cancer. J Clin Oncol 26: 2006-2012.

7. Fuchs CS, Marshall J, Mitchell E, Wierzbicki R, Ganju V, et al. (2007) Randomized, controlled trial of irinotecan plus infusional, bolus, or oral fluoropyrimidines in first-line treatment of metastatic colorectal cancer: results from the BICC-C Study. J Clin Oncol 25: 4779-4786.

8. Hurwitz H, Fehrenbacher L, Novotny W, Cartwright T, Hainsworth J, et al. (2004) Bevacizumab plus irinotecan, fluorouracil, and leucovorin for metastatic colorectal cancer. N Engl J Med 350: 2335-2342.

9. Douillard JY, Siena S, Cassidy J, Tabernero J, Burkes R, et al. (2010) Randomized, phase III trial of panitumumab with infusional fluorouracil, leucovorin, and oxaliplatin (FOLFOX4) versus FOLFOX4 alone as first-line treatment in patients with previously untreated metastatic colorectal cancer: the PRIME study. J Clin Oncol 28: 4697-4705.

10. Van Cutsem E, Peeters M, Siena S, Humblet Y, Hendlisz A, et al. (2007) Openlabel phase III trial of panitumumab plus best supportive care compared with best supportive care alone in patients with chemotherapy-refractory metastatic colorectal cancer. J Clin Oncol 25: 1658-1664.

11. Cunningham D, Humblet $Y$, Siena S, Khayat D, Bleiberg $H$, et al. (2004) Cetuximab monotherapy and cetuximab plus irinotecan in irinotecan-refractory metastatic colorectal cancer. N Engl J Med 351: 337-345.

12. Kemeny N, Huang Y, Cohen AM, Shi W, Conti JA, et al. (1999) Hepatic arteria infusion of chemotherapy after resection of hepatic metastases from colorectal cancer. N Engl J Med 341: 2039-2048.

13. Kemeny N, Conti JA, Cohen A, Campana P, Huang Y, et al. (1994) Phase Il study of hepatic arterial floxuridine, leucovorin, and dexamethasone for unresectable liver metastases from colorectal carcinoma. J Clin Oncol 12: 2288-2295.

14. Kemeny NE, Niedzwiecki D, Hollis DR, Campana P, Huang Y, et al. (2006) Hepatic arterial infusion versus systemic therapy for hepatic metastases from colorectal cancer: a randomized trial of efficacy, quality of life, and molecula markers (CALGB 9481). J Clin Oncol 24: 1395-1403. 
Citation: Meyer JE, Cohen SJ (2011) Beyond First-Line Therapy: Combining Chemotherapy and Radioembolization for Hepatic Colorectal Metastases. J Nucl Med Radiat Ther 2:103. doi:10.4172/2155-9619.1000103

15. Kemeny MM, Adak S, Gray B, et al. Combined-modality treatment for resectable metastatic colorectal carcinoma to the liver: surgical resection of hepatic metastases in combination with continuous infusion of chemotherapy-an intergroup study. J Clin Oncol 20: 1499-1505

16. Riaz A, Kulik LM, Mulcahy MF, Lewandowski RJ, Salem R, et al. (2010) Yttrium-90 Radioembolization in the Management of Liver Malignancies. Semin Oncology 37: 94-101.

17. Pan CC, Kavanaugh BD, Dawson LA, Li XA, Das SK, et al. (2010) Radiationassociated liver injury. Int J Radiation Oncology Biol Phys 76: S94-100.

18. Martinez-Monge R, Nag S, Nieroda CA, Martin EW, (1999) lodine-125 brachytherapy in the treatment of colorectal adenocarcinoma metastatic to the liver. Cancer 85: 1218-1225.

19. Rusthoven KE, Kavanaugh BD, Cardenes H, Stieber VW, Burri SH, et al. (2009) Multi-Institutional Phase I/II Trial of Stereotactic Body Radiation Therapy for Liver Metastases. JCO 27: 1572-1578.

20. Kennedy AS, Nutting C, Coldwell D, Gaiser J, Drachenberg C, et al. (2004) Pathologic response and microdosimetry of $90 \mathrm{Y}$ microspheres in man: review of four explanted whole livers. Int J Radiation Onocology Biol Phys 60: 15521563.

21. Kennedy AS, Salem R (2003) Comparison of two 90Yttrium microsphere agents for hepatic artery brachytherapy. Proceedings of the 14th International Congress on Anti-Cancer Treatment 1: 156.

22. Kennedy A, Nag S, Salem R, Murthy R, McEwan AJ, et al. (2007) Recommendations for Radioembolization of Hepatic Malignancies Using Yttrium-90 Microsphere Brachytherapy: A Consensus Panel Report from the Radioembolization Brachytherapy Oncology Consortium. Int J Radiation Oncology Biol Phys 68: 13-23.

23. Sundermeyer ML, Meropol NJ, Rogatko A, Wang H, Cohen SJ, et al. (2005) Changing patterns of bone and brain metastases in patients with colorectal cancer. Clin Colorectal Cancer 5: 108-113.
24. Tepper J, Krasna MJ, Niedzwiecki D, Hollis D, Reed CE, et al. (2008) Phase III trial of trimodality therapy with cisplatin, fluorouracil, radiotherapy, and surgery compared with surgery alone for esophageal cancer: CALGB 9781. J Clin Oncol 26: 1086-92.

25. Huguet F, André T, Hammel P, Artru P, Balosso J, et al. (2007) Impact of chemoradiotherapy after disease control with chemotherapy in locally advanced pancreatic adenocarcinoma in GERCOR phase II and III studies. J Clin Oncol 25: 326-331.

26. van Hazel GA, Pavlakis N, Goldstein D, Olver IN, Tapner MJ, et al. (2009) Treatment of fluorouracil-refractory patients with liver metastases from colorectal cancer by using yttrium-90 resin microspheres plus concomitant systemic irinotecan chemotherapy. J Clin Oncol 27: 4089-4095.

27. Lim L, Gibbs P, Yip D, Shapiro JD, Dowling R, et al. (2005) A prospective evaluation of treatment with Selective Internal Radiation Therapy (SIR-spheres) in patients with unresectable liver metastases from colorectal cancer previously treated with 5-FU based chemotherapy. BMC Cancer 5: 132

28. Hendlisz A, Van den Eynde M, Peeters M, Maleux G, Lambert B, et al. (2010) Phase III trial comparing protracted intravenous fluorouracil infusion alone or with yttrium-90 resin microspheres radioembolization for liver-limited metastatic colorectal cancer refractory to standard chemotherapy. J Clin Oncol 28: 3687 3694.

29. Chua TC, Bester L, Saxena A, Morris DL (2011) Radioembolization and systemic chemotherapy improves response and survival for unresectable colorectal liver metastases. J Cancer Res Clin Oncol 137: 865-73.

30. Cohen SJ, Konski AA, Putnam S (2011) A phase I study of capecitabine in combination with yttrium-90 labeled resin microspheres (SIR-Spheres) in patients (pts) with advanced cancer. Proc ASCO.

31. FOLFOX Plus SIR-SPHERES MICROSPHERES Versus FOLFOX Alone in Patients With Liver Mets From Primary Colorectal Cancer (SIRFLOX). 